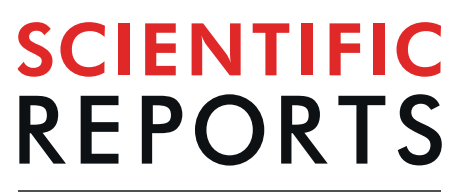

natureresearch

Check for updates

\title{
Association Between Cerebrospinal Fluid Biomarkers and Age-related Brain Changes in Patients with Normal Pressure Hydrocephalus
}

\author{
Foad Taghdiri ${ }^{1,2}$, Melisa Gumus ${ }^{1}$, Musleh Algarni ${ }^{3}$, Alfonso Fasano ${ }^{2,3,4}$, David Tang-Wai ${ }^{2} \&$ \\ Maria Carmela Tartaglia ${ }^{1,2} \bowtie$
}

Our study aimed to: 1)investigate the diagnostic utility of CSF A $\beta 42$, $t$-tau, and $p$-tau to differentiate normal-pressure-hydrocephalus(NPH) from Alzheimer's-disease(AD) and normal-controls; and 2) investigate if age and ventricular size affect the levels of CSF biomarkers in NPH patients. We recruited 131 participants: (a)Suspected-NPH: 72 with ventriculomegaly and clinical symptoms of NPH. These participants were then divided into two groups of 1)Probable-NPH $(\mathrm{N}=38)$ and 2) Unlikely-NPH $(\mathrm{N}=34)$ based on whether participants experienced gait improvement after removal of a large amount of CSF; (b)AD group: 30 participants with CSF biomarkers and cognitive symptoms consistent with $A D$; (c)Control-group: 29 participants who were cognitively and functionally normal. Lower levels of CSF $\mathrm{A} \beta 42$ and $p$-tau were observed in the probable-NPH compared to the normal controls $(444.22 \pm 163.3$ vs. $1213.75 \pm 556.5$; and $26.05 \pm 9.2$ vs. $46.16 \pm 13.3 \mathrm{pg} / \mathrm{mL}$; respectively). Lower levels of CSF p-tau and t-tau were found in the probable-NPH compared to the $A D(26.05 \pm 9.2$ vs. $114.95 \pm 28.2$; and $193.29 \pm 92.3$ vs. $822.65 \pm 311.5 \mathrm{pg} / \mathrm{mL}$; respectively) but the CSF-A 342 was low in both the probable$\mathrm{NPH}$ and AD. CSF-A 342 correlated with age and Evans-index only in the probable- $\mathrm{NPH}(\mathrm{r}=0.460$, $p=0.004$; and $r=-0.530, p=0.001$; respectively). Our study supports the hypothesis that agerelated atrophy results in better $A \beta 42$ clearance in the CSF because of the increase in the interstitial space.

Normal pressure hydrocephalus (NPH) is a syndrome associated with enlarged ventricles without marked elevation in cerebrospinal fluid (CSF) pressure ${ }^{1}$. Clinical symptoms include gait and balance impairment, cognitive deficits, and urinary urgency/incontinence ${ }^{1}$. In both NPH and Alzheimer's disease (AD) decreased CSF levels of the amyloid $\beta-42$ ( $\mathrm{A} \beta 42$ ) have been found; however, in contrast to $\mathrm{AD}$, total tau ( $\mathrm{t}$-tau) and phospho-tau ( $\mathrm{p}$-tau) levels are not increased in NPH cases $^{2,3}$. It has been hypothesized that the low A $\beta 42$ in the presence of low $\mathrm{t}$-tau and p-tau is due to the decrease in interstitial space that precludes amyloid precursor protein (APP) fragments and tau proteins from being effectively cleared by CSF and consequently the levels of these proteins decrease in the $\mathrm{CSF}^{4}$. This hypothesis was based on two major observations: 1) low levels of all APP fragments (i.e., A $\beta 38$, $\mathrm{A} \beta 40, \mathrm{~A} \beta 42$, sAPP $\alpha$, and sAPP $\beta$ ) in CSF obtained both by lumbar and ventricular methods of patients with NPH compared to health controls, which all increased back to normal after shunting ${ }^{1}$ and 2) A $\beta$ clearance from the interstitial fluid is increased during sleep when the size of interstitial space increases by $60 \%{ }^{5}$.

The aims of our study were to: 1) investigate the diagnostic utility of CSF A $\beta 42$, $t$-tau, and p-tau to differentiate $\mathrm{NPH}$ from AD and normal controls; and 2) investigate if age and ventricular size affect the levels of these CSF biomarkers in NPH patients. Answers to these questions would improve understanding of CSF A 342 , t-tau, and p-tau production/clearance and facilitate relating CSF biomarkers to patient characteristics.

\footnotetext{
${ }^{1}$ Tanz Centre for Research in Neurodegenerative Diseases, University of Toronto, 60 Leonard avenue, Toronto, ON, M5T 2S8, Canada. ${ }^{2}$ Department of medicine, Division of Neurology, University of Toronto, Toronto, ON, Canada. ${ }^{3}$ Edmond J. Safra Program in Parkinson's Disease, Morton and Gloria Shulman Movement Disorders Clinic, Toronto Western Hospital, UHN, Toronto, Ontario, Canada. Division of Neurology, University of Toronto, Toronto, Ontario, Canada. ${ }^{4}$ Krembil Brain Institute, Toronto, Ontario, Canada. ${ }^{\bowtie}$-mail: carmela.tartaglia@uhn.ca
} 
a)

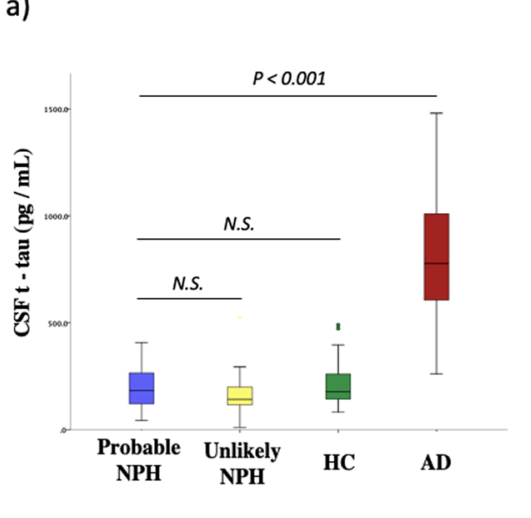

b)

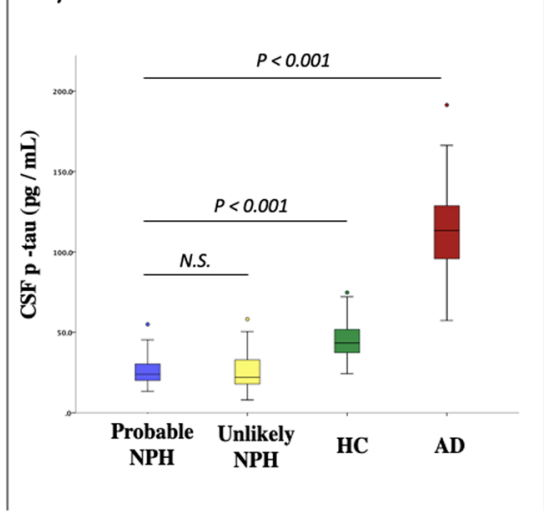

c)

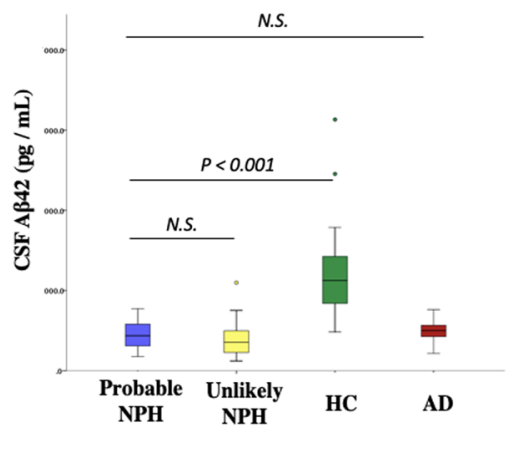

Figure 1. Comparing the levels of CSF biomarkers: (a) t-tau, (b) p-tau, and (c) A $\beta 42$ between the groups (i.e., Probable NPH, unlikely NPH, Normal controls (NC), and Alzheimer's disease (AD).

\begin{tabular}{|l|l|l|l|l|}
\hline \multirow{2}{*}{} & \multicolumn{2}{|l|}{ Suspected NPH } & \multirow{2}{*}{ AD Group } & \multirow{2}{*}{ Control Group } \\
\cline { 2 - 5 } & Probable NPH & Unlikely NPH & 30 & 29 \\
\hline $\mathrm{N}$ & 38 & 34 & $74.06 \pm 7.7$ & $71.9 \pm 5.4$ \\
\hline Age (years) & $73.49 \pm 6.3$ & $70.54 \pm 7.74$ & $30 / 30$ & $0 / 29$ \\
\hline $\begin{array}{l}\text { Abnormal Cognitive } \\
\text { Assessment score }\end{array}$ & $38 / 38$ & $34 / 34$ & $493.20 \pm 139.8$ & $1213.75 \pm 556.5$ \\
\hline CSF A $\beta 42(\mathrm{pg} / \mathrm{mL})$ & $444.22 \pm 163.3$ & $392.61 \pm 213.6$ & $822.65 \pm 311.5$ & $216.27 \pm 107.3$ \\
\hline CSF t-tau $(\mathrm{pg} / \mathrm{mL})$ & $193.29 \pm 92.3$ & $158.46 \pm 92.9$ & $114.95 \pm 28.2$ & $46.16 \pm 13.3$ \\
\hline CSF p-tau $(\mathrm{pg} / \mathrm{mL})$ & $26.05 \pm 9.2$ & $24.67 \pm 11.4$ & & \\
\hline
\end{tabular}

Table 1. Groups description*. *Mean $\pm \mathrm{SD} ;{ }^{\dagger}$ Number of participants in each group with abnormal cognitive (i.e., MoCA, or TorCA) scores.

\section{Results}

Differences in CSF biomarkers between the groups. Average age of the patients in the probable and unlikely NPH groups was not significantly different ( $73.49 \pm 6.3$ vs $70.54 \pm 7.74$ years old; $p=0.13)$. CSF levels of $\mathrm{A} \beta 42$, t-tau, and p-tau were not significantly different between the probable and unlikely NPH groups $(444.22 \pm 163.3$ vs. $392.61 \pm 213.6 \mathrm{pg} / \mathrm{mL}, \mathrm{p}=0.825 ; 193.29 \pm 92.3$ vs. $158.46 \pm 92.9 \mathrm{pg} / \mathrm{mL}, \mathrm{p}=0.514 ;$ and $26.05 \pm 9.2$ vs. $24.67 \pm 11.4 \mathrm{pg} / \mathrm{mL}, \mathrm{p}=0.994$; respectively). However, significantly lower levels of the CSF A $\beta 42$ and p-tau were observed in the probable NPH group compared to the normal controls $(444.22 \pm 163.3$ vs. $1213.75 \pm 556.5 \mathrm{pg} / \mathrm{mL}, \mathrm{p}<0.001$; and $26.05 \pm 9.2 \mathrm{vs}$. $46.16 \pm 13.3 \mathrm{pg} / \mathrm{mL}, \mathrm{p}<0.001$; respectively). When CSF $\mathrm{A} \beta 42$, $\mathrm{t}$-tau and $\mathrm{p}$-tau were compared between the probable NPH group and AD group, lower levels of CSF p-tau and t-tau were found in the probable NPH group compared to the AD group (26.05 $\pm 9.2 \mathrm{vs} .114 .95 \pm 28.2 \mathrm{pg} / \mathrm{mL}$, $\mathrm{p}<0.001$; and $193.29 \pm 92.3$ vs. $822.65 \pm 311.5 \mathrm{pg} / \mathrm{mL}$; respectively) but the mean CSF A 342 was low in both the probable NPH and AD group ( $444.22 \pm 163.3$ vs $493.20 \pm 139.8 \mathrm{pg} / \mathrm{mL}, \mathrm{p}=0.703)$. Figure 1 and Table 1 summarize the levels of CSF biomarkers between the groups.

PCA results. Although the CSF biomarkers could not differentiate probable NPH from unlikely NPH, PCA was able to differentiate the probable NPH group from the $\mathrm{AD}$ and normal controls groups (Fig. 2). Initial eigenvalues indicated that the first three components explained $65 \%, 33 \%$, and $2 \%$ of the variance, respectively. We did not eliminate any of the factors from the PCA analysis since p-tau and t-tau showed factor loading of about 0.7 in the first component while $\mathrm{A} \beta 42$ had - 1.0 factor loading in the second component. We then used the first 2 principal components from the PCA in the SVM to investigate the model predictions in separating the patient groups based on their t-tau, p-tau and A 342 profiles. The SVM predicted the patient category for each individual with a $92 \%$ accuracy $(95 \% \mathrm{CI}=80 \%, 97 \% ; \mathrm{p}<0.001)$ resulting in a sensitivity and specificity of $90 \%$ and $98 \%$, respectively.

Relationship between the CSF biomarkers and age in the probable and unlikely NPH groups. Age was significantly correlated with the levels of CSF A 342 and $p$-tau in the probable NPH group $(r=0.460$, $\mathrm{p}=0.004$; and $\mathrm{r}=0.444, \mathrm{p}=0.006$; respectively) but not in the unlikely NPH group $(\mathrm{r}=0.09, \mathrm{p}=0.674$; and $\mathrm{r}=0.381, \mathrm{p}=0.066$; respectively) or in the normal controls $(\mathrm{r}=-0.221, \mathrm{p}=0.289$; and $\mathrm{r}=0.330, \mathrm{p}=0.107$; respectively) (Fig. 3). Age was not correlated with the levels of CSF t-tau in either of the groups (i.e., probable NPH, unlikely NPH, and normal controls) $(\mathrm{r}=0.298, \mathrm{p}=0.073 ; \mathrm{r}=0.165, \mathrm{p}=0.441 ; \mathrm{r}=0.271, \mathrm{p}=0.191$; respectively).

Relationship between the CSF biomarkers and Evans index in the probable and unlikely NPH groups. Evans index was negatively correlated with the levels of CSF A $\beta 42(r=-0.530, p=0.001)$ in the 


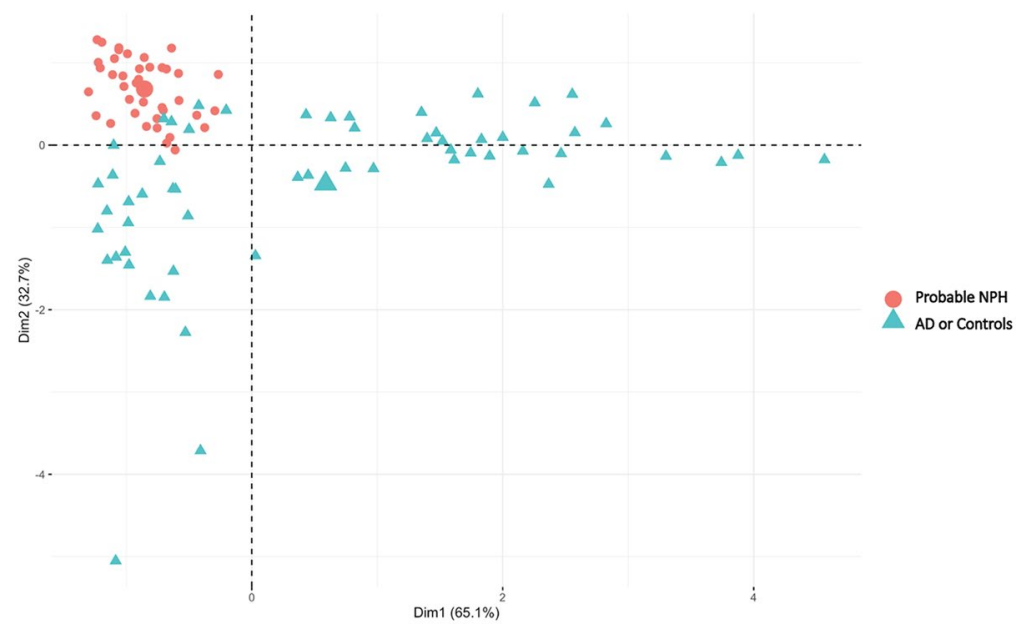

Figure 2. PCA results. Red dots represent probable NPH patients while blue dots represent AD patients or controls.

Black - Normal controls; Red - Probable NPH; Blue - Unlikely NPH
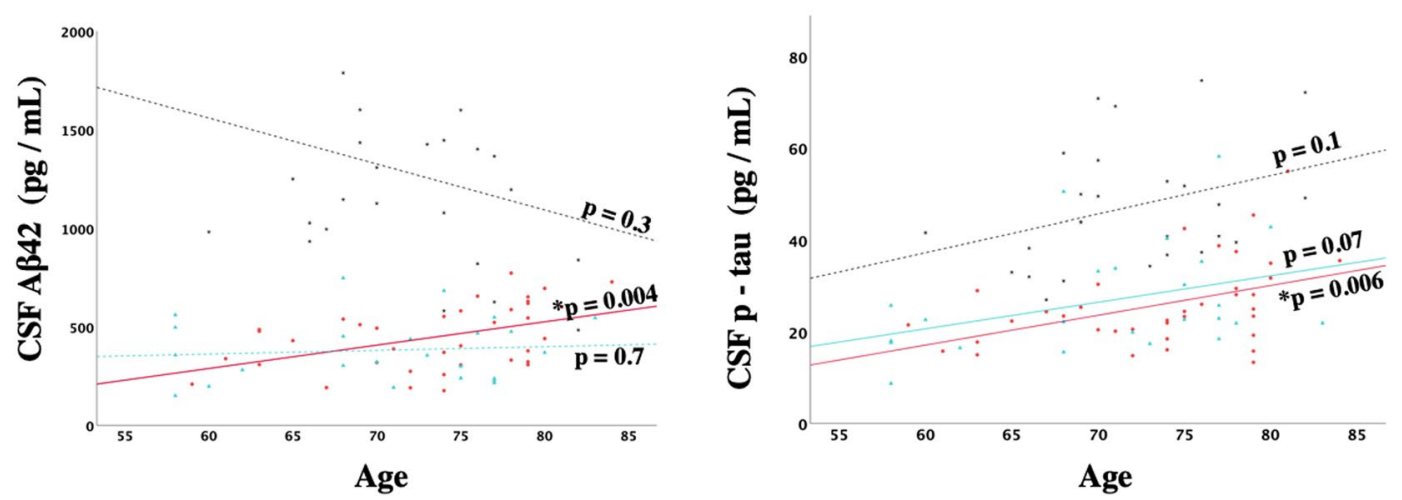

Figure 3. Relationship between CSF biomarkers and age in the probable (red) and unlikely (blue) NPH groups and controls (black).

probable NPH group (Fig. 4). However, no statistically significant correlation was found between the Evans index and the levels of CSF $\mathrm{p}$-tau and $\mathrm{t}$-tau in this group $(\mathrm{r}=-0.229, \mathrm{p}=0.172$; and $\mathrm{r}=-0.049, \mathrm{p}=0.775$; respectively). In the unlikely NPH group, none of the CSF A $\beta 42$, p-tau, and t-tau levels were significantly correlated with the Evans index $(\mathrm{r}=-0.016, \mathrm{p}=0.938 ; \mathrm{r}=0.052, \mathrm{p}=0.799$; and $\mathrm{r}=0.257, \mathrm{p}=0.204$; respectively).

Linear regression model. As mentioned above, the levels of CSF A $\beta 42$ were significantly correlated with both age and Evans index in the probable NPH group. To further assess these relationships, we ran a linear regression analysis entering the CSF A 342 as a dependent variable and age and Evans index as two independent variables. This model could explain $32.6 \%$ of the variability seen in the levels of CSF A $\beta 42(R=0.571, p=0.001)$ and showed that Evans index is contributing to the levels of CSF A $\beta 42$ independent of age (standardized coefficient beta $=-0.398, \mathrm{p}=0.022$ ) in the probable NPH group.

\section{Discussion}

The results of this study revealed lower levels of CSF A 342 and p-tau in the probable NPH group compared to the controls. The mean level of CSF A $\beta 42$ was similar between the probable NPH and AD groups. As our PCA results showed, including CSF A $\beta 42$, p-tau, and t-tau allowed differentiation of probable NPH from controls and patients with $\mathrm{AD}$ (Fig. 2). However, these CSF biomarkers were not significantly different between the probable and unlikely NPH so could not be used to differentiate between these two groups.

The PCA/SVM results (Fig. 2) revealed that CSF A $\beta 42$, p-tau, and t-tau, could be used to differentiate probable $\mathrm{NPH}$ from AD and normal controls with a reasonable sensitivity and specificity (i.e. $90 \%$ and $98 \%$, respectively). However, it should be noted that although a pattern of low CSF A $\beta 42$ level in the presence of low to normal p-tau and t-tau levels in the context of clinical and radiological findings consistent with NPH can increase the likelihood of this disease, it is not pathognomonic. Levels of CSF A $\beta 42$, p-tau, and t-tau failed to differentiate the 


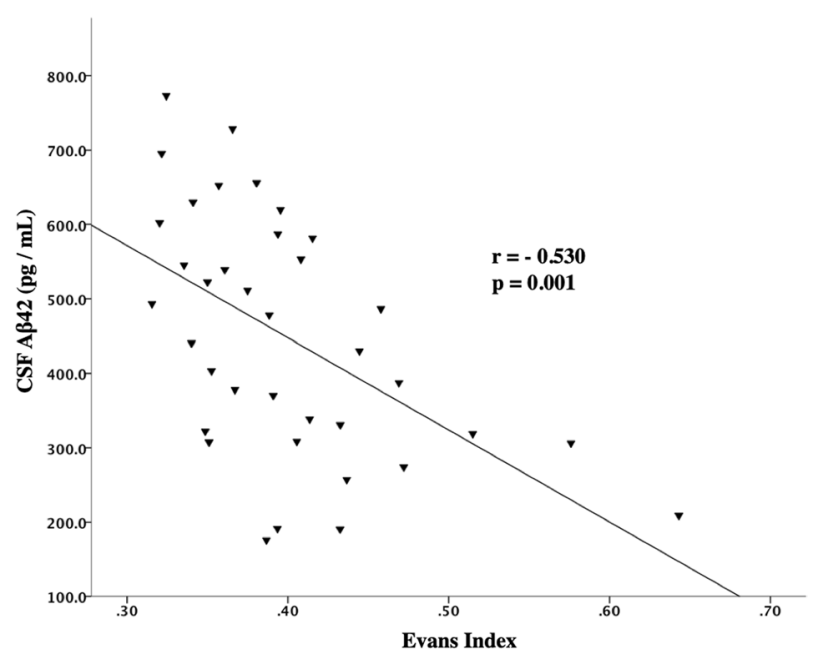

Figure 4. Correlation between CSF A $\beta 42$ levels and Evans index in the probable NPH group.

probable from unlikely NPH in the present study. Although not clear, one possibility is that other comorbidities in the unlikely NPH group are confounding the results.

Interestingly, in the probable NPH group, CSF A 342 was significantly correlated with age and inversely correlated with size of the ventricles, as measured using Evans index. These findings are consistent with the hypothesis that in the setting of $\mathrm{NPH}$, the interstitial space between neurons is decreased and consequently clearance of $\mathrm{A} \beta 42$ into the CSF is impaired ${ }^{4}$. Traditionally, the Evans index has been used as an indirect surrogate marker of ventricular volume in CT and $\mathrm{MRI}^{6,7}$. Larger ventricular volumes, thus higher values of Evans index, in NPH may be associated with less interstitial space. This decreased interstitial space impairs the clearance of A 342 into CSF and may explain the negative correlation observed between the CSF A $\beta 42$ and Evans index. On the other hand, aging is associated with brain atrophy which increases interstitial space and thus allows better clearance of the A $\beta 42$ from the brain. In other words, the natural process of aging and brain atrophy can potentially help with the normalization of the CSF biomarkers in patients with NPH. In addition, these results suggest that patients' characteristics such as their age and the amount of ventriculomegaly should be taken into account before interpreting the CSF biomarkers.

As mentioned above, investigating levels of CSF A 342 , p-tau, and t-tau alone does not suffice to differentiate the probable from unlikely NPH group. However, our results also revealed that CSF A $\beta 42$ is significantly related to the patients' age only in the probable NPH and not in the unlikely NPH group. This raises the question whether longitudinal follow up of CSF A 342 levels rather than measuring them at a single timepoint, may be better for selecting patients with probable NPH. However, more longitudinal studies with large sample sizes are necessary to further investigate this hypothesis. In addition, in this study, we did not investigate the potential causes for the observed decrease in CSF A $\beta 42$ in the probable NPH group (e.g., formation of amyloid plaques, dilution effect, or less production of $\mathrm{A} \beta 42$ ) and more studies are required to investigate this further. Also, it should be noted that as mentioned above, approximately $40 \mathrm{~mL}$ of CSF was collected from each NPH participant. This is a relatively large amount of CSF and may potentially affect the biomarker concentrations. However, large amount CSF collection is common in NPH assessment ${ }^{8}$. The other potential limitation of our study is that we did not measure other possible CSF biomarkers such as A $\beta 40$ or neurofilament light chain. Measuring these biomarkers may potentially help better understand NPH pathophysiology.

In conclusion, our study showed that the mean level of CSF A 342 was similar between the probable NPH and $\mathrm{AD}$ groups and lower than that of normal controls while both $\mathrm{p}$-tau and t-tau levels were significantly lower in probable NPH compared to AD. Also, probable NPH patients with higher A $\beta 42$ values tended to be older in our study which supports the hypothesis that age-related atrophy results in better A $\beta 42$ clearance in the CSF because of the increase in the interstitial space. Finally, given the association between age and levels of CSF A 342 in the probable NPH group, we proposed a hypothesis that following up on the levels of CSF biomarkers longitudinally is a stronger biomarker for the diagnosis of probable NPH compared to a single measurement of these biomarkers.

\section{Methods}

Participants. A total of 131 subjects were included in this study:

(a) Suspected NPH group: 72 patients (age [mean \pm SD], $73.04 \pm 6.5$ years) with ventriculomegaly on MRI brain or CT head with clinical symptoms of NPH (i.e., cognitive impairment, balance/gait impairment, and urinary incontinence) were recruited by three neurologists (MCT, DTW, and AF) through Toronto Western Hospital, University Health Network (UHN), Toronto, Canada. All patients underwent lumbar puncture (LP) and all with a normal opening pressure ${ }^{6}$. Patients with suspected NPH group were divided into two groups based on the criteria previously described by Relkin et al. $\left.{ }^{6}: 1\right)$ Probable NPH: 38 patients with clinical symptoms and radiological findings consistent with NPH who experienced an improvement in their gait after removal of a large amount of CSF (35-40 ml). Large amount of CSF removal caused clinically significant improvement in this 
group's patients' gait. Patients in this group were not suffering from any other neurological, psychiatric, or medical conditions that could potentially explain their presenting symptoms; 2) Unlikely NPH: 34 patients with clinical symptoms similar to the previous group but large amount of CSF removal did not significantly improve their gait impairment, i.e. little or no change in speed or stride length.

(b) AD group: included 30 patients (age, $74.06 \pm 7.7$ years) with CSF biomarkers consistent with $\mathrm{AD}^{9,10}$.

(c) Control group: 29 subjects who were cognitively and functionally normal including gait and neuroimaging but some had a history of depression but no evidence of AD on CSF biomarkers.

Cognitive assessments. As part of the clinical evaluation all participants underwent a cognitive assessment. However, different tests (i.e., Montreal Cognitive Assessment (MoCA) ${ }^{11}$, Toronto Cognitive Assessment $(\text { TorCA })^{12}$ ) were used to assess the cognitive status.

Standard protocol approvals and patient consents. The University Health Network's Research Ethics Board approved the study. Written informed consent was obtained from all participants or their legally authorized representatives, in case the participant was not competent to consent, before participating in the study. All methods were carried out in accordance with relevant guidelines and regulations.

CSF analysis. All subjects underwent a lumbar puncture (LP) that was performed according to Alzheimer's Disease Neuroimaging Initiative (ADNI) protocol ${ }^{13}$. Approximately, $40 \mathrm{~mL}$ of CSF was collected from each NPH participant by allowing CSF to drip into the collecting tubes (gravity drip). After CSF was collected in polypropylene tubes, it was transported to the adjacent laboratory within 30 minutes, aliquoted, and stored at $-80^{\circ} \mathrm{C}$. A sandwich ELISA was used to measure concentrations of $A \beta 42$ (Innotest $\beta$-amyloid (1-42), Fujirebio), p-tau (Innotest phospho-tau (181p), Fujirebio), and t-tau (Innotest hTAU-Ag, Fujirebio) following the manufacturer's instruction ${ }^{14,15}$. All analyses were performed at the Tanz Centre for Research in Neurodegenerative Diseases (Toronto, Ontario) by one operator (FT). All samples were measured in duplicate and repeated if the difference between individual optic density (OD) values was greater than $20 \%$. In addition to the ready-to-use calibrators (CAL) and Run Validation Controls (RVC) which were part of the Fujirebio Innotest assay kits, internal controls were also included in each run. After calculating the mean absorbance for the CAL, RVC, and unknown CSF samples, a sigmoidal 4-parameter curve fitting was used to determine the corresponding concentrations. CSF biomarkers were considered consistent with $\mathrm{AD}$ diagnosis if $\mathrm{p}$-tau $>68 \mathrm{pg} / \mathrm{ml}$ and $\mathrm{A} \beta 42$ to $\mathrm{t}$-tau index (ATI) $<0.8^{9,10}$.

Evans index. Evans index was calculated for the subjects in the suspected NPH group using their axial Fluid-attenuated inversion recovery (FLAIR) magnetic resonance images (MRI) or axial computed tomography (CT) images when MRI was not available. The ratio of the maximum width of the frontal horns of the lateral ventricles to the maximal internal diameter of the skull at the same slice of the axial MRI or CT images was used to calculate this index ${ }^{16}$.

Principle component analysis (PCA). We constructed a Support Vector Machine (SVM) to separate the probable NPH patients from AD and controls based on individual levels of CSF A 342 , $t$-tau and p-tau. SVM is a powerful supervised learning algorithm that can be trained on a subset of data and test its predictions on the rest of the testing data. First, a Principle Component Analysis (PCA) was used to visualize the data and compute composite scores for $\mathrm{A} \beta 42$, $\mathrm{t}$-tau and $\mathrm{p}$-tau for each patient. The first two components of PCA were then used in the SVM for determining whether the linear combination of CSF A $\beta 42, t$-tau, $p$-tau levels separated probable $\mathrm{NPH}$ patient from the other two groups (i.e., AD and controls). The SVM was designed with a linear kernel for the classification, and we used 8-fold cross validation that was implemented in the function. PCA in this process was not used to select the significant factors, but rather utilized to create composite variables as an input for SVM. For this step, all data was scaled and processed using R studio ${ }^{17}$.

Statistical analysis. Statistical analyses were conducted using SPSS software (SPSS Inc. v. 24). One-way analysis of variance (ANOVA) was used to compare the CSF biomarkers (i.e., A 342 , $\mathrm{t}$-tau and p-tau) among the groups (i.e., probable NPH, unlikely NPH, AD, NC). Dunnett-T3 post hoc analysis ${ }^{18}$ was performed to compare the probable NPH group with each of the 3 other groups. The Pearson correlation coefficient was used for correlations. Statistical significance level was set at $\mathrm{p}<0.05$.

\section{Data availability}

All data can be shared at the request of other investigators.

Received: 31 March 2020; Accepted: 16 May 2020;

Published online: 04 June 2020

\section{References}

1. Jeppsson, A., Zetterberg, H., Blennow, K. \& Wikkelsø, C. Idiopathic normal-pressure hydrocephalus: pathophysiology and diagnosis by CSF biomarkers. Neurology 80, 1385-1392 (2013).

2. Kapaki, E. et al. Cerebrospinal fluid tau, phospho-tau 181 and $\beta$-amyloid $1-42$ in idiopathic normal pressure hydrocephalus: a discrimination from Alzheimer's disease. European journal of neurology 14, 168-173 (2007)

3. Ågren-Wilsson, A. et al. CSF biomarkers in the evaluation of idiopathic normal pressure hydrocephalus. Acta neurologica Scandinavica 116, 333-339 (2007)

4. Graff-Radford, N. R. Alzheimer CSF biomarkers may be misleading in normal-pressure hydrocephalus. Neurology 83, 1573-1575 (2014).

5. Xie, L. et al. Sleep drives metabolite clearance from the adult brain. science 342, 373-377 (2013). 
6. Relkin, N., Marmarou, A., Klinge, P., Bergsneider, M. \& Black, P. M. Diagnosing idiopathic normal-pressure hydrocephalus. Neurosurgery 57, S2-4 (2005).

7. Synek, V., Reuben, J. \& Du Boulay, G. Comparing Evans' index and computerized axial tomography in assessing relationship of ventricular size to brain size. Neurology 26, 231-231 (1976).

8. Sand, T. et al. Idiopathic normal pressure hydrocephalus: the CSF tap-test may predict the clinical response to shunting. Acta neurologica scandinavica 89, 311-316 (1994).

9. Blennow, K. Cerebrospinal fluid protein biomarkers for Alzheimer's disease. NeuroRx 1, 213-225 (2004).

10. Blennow, K. et al. Clinical utility of cerebrospinal fluid biomarkers in the diagnosis of early Alzheimer's disease. Alzheimer's \& Dementia 11, 58-69 (2015).

11. Nasreddine, Z. S. et al. The Montreal Cognitive Assessment, MoCA: a brief screening tool for mild cognitive impairment. Journal of the American Geriatrics Society 53, 695-699 (2005).

12. Freedman, M. et al. The Toronto Cognitive Assessment (TorCA): normative data and validation to detect amnestic mild cognitive impairment. Alzheimer's research \& therapy 10, 65 (2018).

13. Jack, C. R. Jr et al. The Alzheimer's disease neuroimaging initiative (ADNI): MRI methods. Journal of Magnetic Resonance Imaging: An Official Journal of the International Society for Magnetic Resonance in Medicine 27, 685-691 (2008).

14. Hulstaert, F. et al. Improved discrimination of AD patients using $\beta$-amyloid (1-42) and tau levels in CSF. Neurology 52, 1555-1555 (1999).

15. Maddalena, A. et al. Biochemical diagnosis of Alzheimer disease by measuring the cerebrospinal fluid ratio of phosphorylated tau protein to $\beta$-amyloid peptide42. Archives of neurology 60, 1202-1206 (2003).

16. Toma, A. K., Holl, E., Kitchen, N. D. \& Watkins, L. D. Evans' index revisited: the need for an alternative in normal pressure hydrocephalus. Neurosurgery 68, 939-944 (2011).

17. Team, R. C. R: A Language and Environment for Statistical Computing. (2018).

18. Shingala, M. C. \& Rajyaguru, A. Comparison of post hoc tests for unequal variance. International Journal of New Technologies in Science and Engineering 2, 22-33 (2015).

\section{Acknowledgements}

We'd like to thank all participants for generous donation of their time to participate in this research program. Toronto General and Western Hospital Foundation; There was no role of the funders for this study.

\section{Author contributions}

F.T. and M.C.T. wrote the main manuscript, helped recruiting patients, did the analyses, prepared figures and tables. M.G. and M.A. helped with statistical analysis, preparing figures and manuscript writing. M.A., A.F., and D.T. helped with patient recruitment, and manuscript concept and design.

\section{Competing interests}

F Taghdiri reports no disclosures. M Gumus reports no disclosures. M Algarni reports no disclosures. D TangWai reports no disclosures. MC Tartaglia reports no disclosures. A Fasano received honoraria for consultancies from Abbvie, Abbott, BrainLab, Boston Scientific, Chiesi farmaceutici, Ipsen, Medtronic, Sunovion, and UCB; honaria for participation in advisory boards from Abbvie, Boston Scientific, and Ipsen; research grants from Abbvie, Boston Scientific, Cummings Foundation, Dystonia Medical Research Foundation Canada, Michael J. Fox Foundation, Medtronic, University of Toronto, and Weston foundation.

\section{Additional information}

Correspondence and requests for materials should be addressed to M.C.T.

Reprints and permissions information is available at www.nature.com/reprints.

Publisher's note Springer Nature remains neutral with regard to jurisdictional claims in published maps and institutional affiliations.

(c) (i) Open Access This article is licensed under a Creative Commons Attribution 4.0 International

License, which permits use, sharing, adaptation, distribution and reproduction in any medium or format, as long as you give appropriate credit to the original author(s) and the source, provide a link to the Creative Commons license, and indicate if changes were made. The images or other third party material in this article are included in the article's Creative Commons license, unless indicated otherwise in a credit line to the material. If material is not included in the article's Creative Commons license and your intended use is not permitted by statutory regulation or exceeds the permitted use, you will need to obtain permission directly from the copyright holder. To view a copy of this license, visit http://creativecommons.org/licenses/by/4.0/.

(C) The Author(s) 2020 